\title{
Les traitements thermiques appliqués aux produits laitiers et leurs conséquences dans le domaine azoté
}

\author{
par \\ Jean ADRIAN \\ Centre de Recherches sur la nutrition, C.N.R.S. - 92190 Bellevue
}

\section{INTRODUCTION}

Les conséquences des traitements technologiques et des modifications survenant lors de la conservation prennent une importance croissante dans le domaine nutritionnel étant donné la part de plus en plus grande des produits alimentaires passant par un cycle industriel.

Les risques d'altération des facteurs vitaminiques ont fait l'objet d'une multitude d'observations qui sont rassemblées périodiquement dans des revues d'ensemble concernant l'évolution des vitamines dans les produits laitiers ainsi que dans les autres matières alimentaires traitées industriellement (Causeret et Mocquot, 1964 ; Causeret et al., 1970 ; Adrian et Petit, 1970, etc.).

L'autre domaine sensible à de nombreux processus technologiques, notamment aux chauffages, est celui des protéines qui sont d'abord dénaturées, ensuite endommagées par la réaction de Maillard.

Celle-ci fait partie des phénomènes de brunissement non enzymatique et risque de se développer chaque fois que des protéines ou des acides aminés sont mis en présence de sucres à fonction réductrice (aldoses, cétoses). Pratiquement, soit au cours des traitements thermiques, soit au cours de conservations prolongées en atmosphère relativement humide, tous les produits alimentaires peuvent subir une réaction de Maillard.

Cette réaction se traduit par des modifications de la présentation physique du produit, de sa qualité organoleptique et nutritionnelle (Adrian, 1972). Dans certains cas, les avantages qui en résultent peuvent apparaître plus importants que les dommages qu'elle provoque. Tel est le cas, par exemple, des dérivés céréaliers (croûte de pain, biscuits, biscottes) dont la valeur commerciale est accrue par un brunissement et un arôme appétents. Comme les céréales n'ont, par ailleurs, qu'une qualité protidique moyenne les préjudices 
nutritionnels enregistrés après un grillage ne peuvent suffire à condamner ce type de technologie.

Le bénéfice résultant d'une réaction de Maillard bien conduite est encore plus apparent dans d'autres domaines : le développement de l'arôme de l'arachide grillée ou de la fève de cacao grillée est imputable en grande partie sinon en totalité à des réactions entre acides aminés et sucres libres.

La situation est entièrement différente avec les produits laitiers. Les composants du lactosérum - protéines solubles et lactose sont très sensibles aux traitements thermiques et une réaction de Maillard se décèle très rapidement dans un tel milieu.

Les conséquences des chauffages sont alors multiples et affectent divers aspects :

- un brunissement fait perdre aux produits laitiers leur qualité commerciale,

- un blocage et une destruction rapide de la lysine en diminue l'intérêt protidique dans une proportion importante,

- les produits formés au cours des chauffages peuvent retentir défavorablement aussi bien sur la valeur organoleptique du lait que sur la qualité hygiénique.

Au total, dans le lait, les traitements thermiques et la réaction de Maillard constituent des phénomènes indiscutablement néfastes dès qu'ils dépassent une certaine intensité. Leur connaissance détaillée peut permettre à la fois de préciser les risques encourus et d'en prévenir l'apparition. C'est pourquoi l'étude de la réaction de Maillard dans le cas des produits laitiers doit intéresser aussi bien le monde industriel et technologique que le nutritionniste et l'hygiéniste.

\section{MODIFICATIONS PHYSICO-CHIMIQUES}

Les premières répercussions d'un traitement thermique - même modéré - concernent la structure et les propriétés physiques des protéines du lactosérum et de la caséine : on assiste à une dénaturation des protéines solubles tandis que la caséine tend à se déstructurer si le traitement est suffisamment intense. De tels phénomènes sont susceptibles de se produire durant des chauffages industriels variés : pasteurisation classique, sterilisation par la technique UHT, etc. Dans tous les cas, la dénaturation des protéines du sérum survient beaucoup plus facilement et avec une intensité beaucoup plus forte que la précipitation de la caséine.

\section{Dénaturation des protéines du lactosérum}

La dénaturation des protéines solubles consiste en une modification de leur structure qui leur fait perdre leurs propriétés électrophorétiques. 
En ce qui concerne la $\beta$ lactoglobuline, la dénaturation se déroule en deux étapes : la molécule - initialement sous forme de dimère se dissocie et se présente à l'état de monomère puis, dans un deuxième temps, on assiste à un déploiement de la chaîne moléculaire. Ce dernier phénomène est dû à des modifications chimiques concernant particulièrement les ponts sulfurés.

Le degré de dénaturation des protéines dépend à la fois de la durée et de l'intensité du traitement thermique. Ainsi, un chauffage à $74^{\circ} \mathrm{C}$ dénature $10 \mathrm{p} .100$ des protéines du sérum s'il se poursuit pendant $2 \mathrm{mn}$ (Harland et al., 1952) mais il touche 50 p. 100 des protéines après $30 \mathrm{mn}$ (Tagari et Roy, 1969).

Corollairement, un chauffage d'une durée uniforme de 2 s dénature 8 p. 100 de l'azote du sérum s'il est effectué à $93^{\circ} \mathrm{C}$ et 57 p. 100 si le traitement a lieu à $143^{\circ} \mathrm{C}$ (Melachouris et Tuckey, 1966).

On estime qu'une dénaturation totale des protéines du sérum est obtenue après un chauffage de $1 \mathrm{~h}$ à $77^{\circ} \mathrm{C}$ ou après $30 \mathrm{mn}$ à $90^{\circ} \mathrm{C}$.

Cependant, le couple durée-intensité du traitement ne constitue pas une donnée suffisante pour prévoir avec précision le comportement des protéines solubles. Par exemple, dans les traitements UHT, la technique indirecte utilisant des échangeurs de chaleur est plus préjudiciable que le procédé direct faisant appel à l'injection de vapeur (tab. 1). Il est fort probable que la plus grande dénaturation enregistrée avec les échangeurs de chaleur résulte de points de surchauffe inhérents à ce genre d'appareillage.

Par ailleurs, le $\mathrm{pH}$ du lactosérum peut déterminer largement le comportement de ses constituants: lorsqu'un traitement de $30 \mathrm{mn}$ à $82^{\circ} \mathrm{C}$ et à $\mathrm{pH} 3,5$, n'entraîne aucune modification physico-chimique, la même opération conduite avec un lactosérum à la neutralité est responsable de la dénaturation de 80 p. 100 des protéines (Guy et al., 1967).

Sur le plan chimique, les phénomènes de dénaturation s'accompagnent de remaniements et de rupture des groupements sulfhydryles et des disulfures. Comme ces groupes se trouvent concentrés dans les protéines du sérum et, accessoirement, dans la caséine $K$, les effets des traitements thermiques porteront principalement sur ces fractions protidiques.

Toutes les fractions protidiques du sérum n'offrent pas une sensibilité uniforme aux chauffages (*), ainsi qu'il apparaît dans les résultats présentés dans les tableaux 1 et 2 . Selon Damicz et al. (1965), Lontie et Préaux (1967), Zadow (1969), et d'autres, les albumines résistent bien à la dénaturation; à l'inverse, dans les

(*) Non seulement chaque type de protéine présente un comportement propre à la dénaturation, mais il en est de même de chaque variant génétique (Gough et Jenness, 1962 ; Dupont, 1965). 


\section{TABLEAU 1}

Répartition des protéines du sérum selon le mode et l'intensité du traitement UHT ${ }^{*}$ )

(en pourcentage de la composition du lait cru)

\begin{tabular}{|c|c|c|c|c|c|c|c|c|}
\hline \multirow{3}{*}{ Fraction du lait } & \multicolumn{8}{|c|}{ Intensité et modalités du chauffage $(* *)$} \\
\hline & \multicolumn{2}{|c|}{$95^{\circ} \mathrm{C}$} & \multicolumn{2}{|c|}{$125^{\circ} \mathrm{C}$} & \multicolumn{2}{|c|}{$145^{\circ} \mathrm{C}$} & \multicolumn{2}{|c|}{$\begin{array}{c}\text { Dénaturation } \\
\text { moyenne }\end{array}$} \\
\hline & échangeur & vapeur & échangeur & vapeur & échangeur & vapeur & échangeur & vapeur \\
\hline Sérum total & -46 & -27 & -69 & -43 & -81 & -53 & 65 & 41 \\
\hline Albumines totales & -55 & -34 & -74 & -40 & -88 & -46 & 72 & 40 \\
\hline$\beta$ lactoglobulines & -66 & -20 & -88 & -30 & -92 & -41 & 82 & 30 \\
\hline Protéose-peptones & -15 & -12 & -30 & -51 & -32 & -70 & 26 & 44 \\
\hline Globulines & -30 & -4 & -72 & -49 & -86 & -75 & 63 & 43 \\
\hline
\end{tabular}

(*) D'après Zadow (1969).

${ }^{(* *}$ Comparaison d'un traitement de $3 \mathrm{~s}$ dans lequel le chauffage se fait indirectement par l'emploi d'un échangeur à plaques ou directement par injection de vapeur. 
valeurs du tableau 2, les albumines paraissent nettement plus sensibles que les autres fractions azotées puisque dans le cas d'une dénaturation moyenne de 30 p. 100 de l'azote non-caséine, les albumines sont dénaturées à raison de 57 p. 100 .

Quant à prévoir le degré de dénaturation résultant d'une opération industrielle, il s'agit d'une estimation difficile en raison de la multiplicité des variantes possibles pour une même opération : la pasteurisation peut être conduite à des températures différentes, un traitement UHT ou un séchage s'effectue à des températures qui varient d'une installation à l'autre et, de surcroît, s'accompagne de pré-chauffages dont les modalités ne sont pas codifiées.

Pour tenter néanmoins d'illustrer les conséquences des divers traitements classiques appliqués au lait, nous rassemblons dans le tableau 2 les répercussions de diverses opérations industrielles en précisant pour chacune les modalités exactes dans lesquelles elles se déroulent. Les valeurs obtenues ne s'appliquent évidemment qu'aux produits traités dans des conditions identiques.

L'importance du processus exact de l'opération apparaîtra immédiatement à l'examen des poudres de laits atomisées après un pré-traitement d'intensité variable : selon la nature du pré-chauffage, la dénaturation moyenne de la poudre de lait oscille dans un rapport de 1 à 5 .

Quoiqu'il en soit, les données du tableau 2 confirment bien le rôle primordial de la sévérité du traitement thermique dans les phénomènes de dénaturation des protéines du sérum.

Cette dénaturation affecte-t-elle la valeur nutritionnelle ? Il ne semble pas que des études parfaitement démonstratives aient été menées dans ce sens ; il faut souhaiter que les nouvelles techniques de séparation puissent permettre de travailler avec des protéines purifiées de sérum, natives ou dénaturées.

Dans l'état actuel des connaissances, on peut considérer que la dénaturation n'affecte pas l'efficacité nutrionnelle globale (digestibilité, valeur biologique) des protéines du lait. Telle est, notamment, l'opinion de Porter (1966) qui est renforcée par l'expérimentation de Lang et al. (1965) dont les résultats peuvent se condenser ainsi :

\begin{tabular}{l|c|c|c|c}
\hline & $\begin{array}{c}\text { Lait } \\
\text { cru }\end{array}$ & $\begin{array}{c}\text { Lait } \\
\text { pasteurisé }\end{array}$ & $\begin{array}{c}\text { Lait } \\
\text { stérilisé }\end{array}$ & $\begin{array}{c}\text { Lait } \\
\text { upérisé }\end{array}$ \\
\hline Dénaturation (p. 100) & 0 & 13 & 53 & 40 \\
C.E.P. ${ }^{*}$ ) & 3,30 & 3,32 & 3,28 & 3,38 \\
\hline
\end{tabular}

${ }^{*}$ ) Coefficient d'Efficacité Protidique = gain de poids / protéines ingérées. 
TABLEAU 2

Répartition des fractions azotées des laits transformés (*) (en pourcentage de la composition du lait cru)

\begin{tabular}{|c|c|c|c|c|c|c|}
\hline \multirow[b]{2}{*}{ Fraction du lait } & \multicolumn{3}{|c|}{ Laits liquides } & \multicolumn{3}{|c|}{ Laits en poudre } \\
\hline & $\begin{array}{c}\text { pasteurisé } \\
\text { (a) }\end{array}$ & $\begin{array}{l}\text { upérisé } \\
\text { (b) }\end{array}$ & $\begin{array}{l}\text { stérilisé } \\
\text { (c) }\end{array}$ & $\begin{array}{l}\text { atomis } \\
\text { pré-chauffage } \\
\text { court } \\
\text { (d) }\end{array}$ & $\begin{array}{l}\text { après } \\
\text { pré-chauffage } \\
\text { long } \\
\text { (e) }\end{array}$ & $\begin{array}{l}\text { Séché } \\
\text { sur } \\
\text { cylindre } \\
\text { (f) }\end{array}$ \\
\hline $\begin{array}{l}\text { Caséine } \\
\text { Non-caséine } \\
\text { Sérum total } \\
\text { Protéose-peptone + globulines } \\
\text { Albumines totales } \\
\beta \text { lactoglobuline } \\
\text { Autres lactalbumines }\end{array}$ & $\begin{array}{l}+4 \\
-13 \\
-21 \\
-15 \\
-22 \\
-29 \\
+31\end{array}$ & $\begin{array}{l}+11 \\
-39 \\
-57 \\
-23 \\
-64 \\
-73 \\
-47\end{array}$ & $\begin{array}{l}+15 \\
-53 \\
-78 \\
-15 \\
-90 \\
-98 \\
-76\end{array}$ & $\begin{array}{r}0 \\
0 \\
-\quad 3 \\
-11 \\
-15 \\
-18 \\
-\quad 9\end{array}$ & $\begin{array}{l}+9 \\
-32 \\
-48 \\
-27 \\
-63 \\
-59 \\
-69\end{array}$ & $\begin{array}{l}+12 \\
-42 \\
-63 \\
-13 \\
-86 \\
-96 \\
-67\end{array}$ \\
\hline Dénaturation moyenne & 11 & 50 & 68 & 9 & 50 & 61 \\
\hline
\end{tabular}

(*) D'après Hostettler et al. (1965) et Shillam et Roy (1963).

$\mathrm{a}=$ pasteurisation à $85^{\circ} \mathrm{C}$ par circulation.

$\mathrm{b}=$ pré-chauffage à $80^{\circ} \mathrm{C}$, upérisation $2,4 \mathrm{~s}$ à $150^{\circ} \mathrm{C}$ et refroidissement à $80^{\circ} \mathrm{C}$.

$\mathrm{c}=116^{\circ} \mathrm{C}$ pendant $45 \mathrm{mn}$.

$\mathrm{d}=$ pré-chauffage de $15 \mathrm{~s}$ à $74^{\circ} \mathrm{C}$.

$\mathrm{e}=$ pré-chauffage de $30 \mathrm{mn}$ à $77^{\circ} \mathrm{C}$.

$\mathrm{f}=$ pré-chauffage à $110^{\circ} \mathrm{C}$ et pré-condensation pour amener à $23 \mathrm{p} .100$ de mat. sèche, puis séchage sur rouleau à $116^{\circ} \mathrm{C}$. 
Par souci d'objectivité, il convient néanmoins de mentionner d'anciens travaux cités par Shillam et al. (1962) tendant à montrer que les traitements aboutissant à une dénaturation partielle des protéines du sérum pouvaient affecter légèrement la digestibilité ou l'efficacité métabolique des protéines lactiques. En réalité, ces observations datent d'une période où les relations " traitements technologiques - valeur nutritionnelle » étaient encore très mal définies ; on peut imaginer que les modifications enregistrées aient pu être faussement attribuées à une dénaturation des protéines.

Enfin, si l'utilisation globale ne semble pas perturbée par la dénaturation, nous ne connaissons apparemment pas le comportement des protéines dénaturées au cours de la protéolyse digestive et la cinétique de leur utilisation.

\section{Modifications de la caséine}

Les caséines sont plus stables thermiquement que les protéines du sérum : ainsi, un chauffage de $10 \mathrm{mn}$ à $88^{\circ} \mathrm{C}$ ne modifie ni la taille, ni la composition des micelles de caséine (Morr, 1965). Cependant, sous l'effet de traitements plus intenses les caséines se révèlent sujettes à des modifications de structure et de composition.

La première manifestation de ces changements est une augmentation apparente de la masse de caséine, due au passage des protéines dénaturées solubles dans la phase colloïdale (Shillam et al., 1960 ; Hostettler et al., 1965 b) :

Azote total

Azote de la caséine

Azote de la fraction non-caséine

Azote des protéines du sérum

Somme caséine + sérum

\begin{tabular}{|c|c|c|c}
\multicolumn{2}{c|}{ Lait cru } & \multicolumn{2}{|c}{ Lait UHT } \\
\cline { 2 - 3 } mgN/100 ml & $\begin{array}{c}\text { p. } 100 \\
\text { de N total }\end{array}$ & mg N/100 ml & $\begin{array}{c}\text { p.100 } \\
\text { de N total }\end{array}$ \\
\cline { 2 - 3 } 534,5 & 100 & 530,0 & 100 \\
420,0 & 78,5 & 465,0 & 88 \\
124,0 & 23 & 64,5 & 12 \\
92,5 & 17,5 & 32,0 & 6 \\
512,5 & 96 & 497,0 & 94 \\
& & & \\
\hline
\end{tabular}

Les protéines du sérum se complexent sélectivement avec la caséine $\alpha$ : le poids de cette fraction augmente de 20 p. 100 après 
stérilisation $\left(^{*}\right)$ tandis que celui de la caséine $\beta$ demeure inchangé (Murthy et al., 1958). C'est la raison pour laquelle le rapport " caséine $\alpha$ / caséine $\beta$ " augmente avec l'intensité du traitement thermique : la stérilisation accroît cette valeur de 42 p. 100 mais la pasteurisation et même l'upérisation demeurent pratiquement sans effet (Hostettler et al., 1965).

Ces observations traduisent essentiellement une interaction entre la $\beta$ lactoglobuline et la caséine $\mathrm{K}$ qui se complexent par l'intermédiaire de réactions entre des fonctions sulfhydryles et grâce à l'intervention du calcium (Rose, 1962 ; Morr et Josephson, 1968 ; Sawyer et al., 1963).

Si le chauffage est suffisamment intense, la taille des micelles et des particules de caséine se modifient dans une double direction.

D'une part, une petite fraction des micelles se désagrège et n'est plus sédimentable après un traitement UHT ou une stérilisation : le lait cru ne renferme que 5 p. 100 de caséine non récupérable par ultracentrifugation tandis que ce pourcentage est de 9 p. 100 après une pasteurisation, de 17 p. 100 environ après une upérisation et de l'ordre de 30 p. 100 après une stérilisation (Hostettler et al., 1965 b ; Morr, 1969).

De l'autre, les micelles non-désagrégées tendent à augmenter de volume. Là aussi il faut des traitements plus sévères que la pasteurisation pour enregistrer des modifications appréciables (Hostettler et al., 1965 b ; Fox et al., 1967 ; Morr, 1969). Les variations de taille-s'observent au microscope électronique ou se mesurent par le coefficient de sédimentation. Pour fixer un ordre de grandeur, les $3 / 4$ des micelles non-détruites ont un diamètre de l'ordre de 50 à $100 \mathrm{~nm}$ dans le lait cru, de 100 à $150 \mathrm{~nm}$ dans le lait stérilisé et de 125 à $175 \mathrm{~nm}$ dans le lait UHT. Dans ce cas, le phénomène est surtout sous la dépendance de l'intensité du chauffage et non de la somme de chaleur appliquée.

Les effets thermiques portent sélectivement sur certaines fractions de la micelle : la caséine $\beta$ se révèle plus stable que la fraction $\alpha$. A l'intérieur de cette fraction, la caséine $K$ offre une grande sensibilité et se trouve protégée par la fraction $\alpha$ s (Swirski et al., 1962 ; Zittle, 1969).

En faisant varier les proportions respectives des diverses caséines, Schmidt (1969) observe que les caséines $\alpha_{\text {s }}$ et $\beta$ tendent à provoquer un accroissement des particules lors des traitements thermiques, tandis que la caséine $K$ est responsable des phénomènes de désagrégation. Ces mécanismes s'apparenteraient à ceux de la coalescence,

(*) Au couns de cet exposé, le terme stérilisation s'appliquera à la technique classique consistant en un traitement de $1^{\prime}$ ordre de $120^{\circ} \mathrm{C}$ pendant une vingtaine de minutes. Ceux faisant appel à des températures nettement plus élevées sont désignés sous le vocable de traitement UHT. 
dans la mesure où l'intérieur d'une micelle se compose essentiellement de caséine $\beta$ et $\alpha_{\mathrm{s}}$, la surface étant constituée en majeure partie de caséine K (Payens, 1966).

Kirchmeier (1962), rattache le comportement thermique des caséines à leur teneur en cystéine et cystine. Cette théorie expliquerait la sensibilité de la caséine $\alpha$ par rapport à la fraction $\beta$, la première renfermant cinq fois plus de cystine que la seconde (Adrian, 1973).

Outre les modifications de structure, les traitements thermiques sont responsables de transformations chimiques des caséines et, dans ce domaine également, la fraction $\alpha$ est la plus sensible.

Sous l'effet de la stérilisation, la caséine $\mathrm{K}$ est susceptible de se décomposer en une para-caséine-chaleur et en glycopeptides (Alais et al., 1966, 1967). Les produits obtenus par cette voie sont très proches sinon identiques à ceux résultant de l'action de la présure. Notamment, dans les deux cas, les glycopeptides renferment de l'acide $\mathrm{N}$ acétyl neuraminique, du galactose et du deoxy-galactose (Hindle et Wheelock, 1970). Les quantités de glycopeptides formés sont proportionnelles à la durée et à l'intensité du traitement thermique.

Par ailleurs, les chauffages provoquent une déphosphorylation des caséines. Ainsi, au cours de la stérilisation le taux de phosphore non-protidique de la caséine entière et de la fraction $K$ est multiplié par 5 , tandis que celui de la caséine $\alpha_{\text {s }}$ est augmenté de 8,5 fois. A l'inverse, la caséine $\beta$ demeure pratiquement inchangée dans ces conditions (Alais et al., 1966 ; Swirski et al., 1962).

La libération du phosphore de la caséine totale est de l'ordre de 50 p. 100 dans une solution de caséine chauffée 1 h à $120^{\circ} \mathrm{C}$ et atteint 99 p. 100 après $1 \mathrm{~h}$ à $140^{\circ} \mathrm{C}$ (Nakanishi et Itoh, 1967).

Dans une certaine mesure, une perte de calcium peut être enregistrée dans la micelle, mais ce phénomène est de moindre ampleur que les risques d'insolubilisation du calcium présent dans la phase aqueuse (Shillam et al., 1960 ; Fox et al., 1967). Des phénomènes plus accentués s'observent dans des laits évaporés ou concentrés, notamment au cours des périodes de conservation, avec des insolubilisations d'éléments azotés et minéraux (Fox et al., 1967 ; Morr, 1965). Les mécanismes mis en jeu sont nettement différents de ceux qui nous intéressent présentement et, par conséquent, ce genre d'évolution ne sera pas traité ici.

Les modifications de structure de la caséine, analogues à la dénaturation des protéines du sérum, retentissent-elles sur l'efficacité protidique de ces molécules ? Nous ne disposons pratiquement pas d'éléments de réponse.

Dans ce domaine on peut citer les essais in vitro conduits par Swirski et al. (1962) mettant en relief que certaines fractions de caséine résistent à l'hydrolyse trypsique après leur chauffage : 


\begin{tabular}{l|c|c}
\hline & $\begin{array}{c}\text { Pourcentage d'azote libéré dans la caséine } \\
\text { témoin }\end{array}$ & chauffée $40 \mathrm{mn}$ à $120^{\circ} \mathrm{C}$ \\
\cline { 2 - 3 } Caséine totale & 15,5 & 6,5 \\
Caséine $\alpha$ & 21,5 & 9,5 \\
Caséine $\beta$ & 9,0 & 9,0 \\
\hline
\end{tabular}

Il paraît très hasardeux de vouloir tirer de ces observations des conclusions relatives à l'efficacité physiologique des caséines dénaturées par les traitements thermiques.

\section{MODIFICATIONS CHIMIQUES :}

\section{la réaction de Maillard}

Les transformations physiques - dénaturation et destructuration décrites jusqu'à maintenant, s'observent surtout dans les laits liquides et les solutions, c'est-à-dire dans les milieux fortement hydratés.

Ceux-ci protègent le lait contre les réactions chimiques en général et réduisent en particulier les risques d'une réaction de Maillard. Pour qu'une telle réaction se développe de manière optimale il convient, en effet, que l'hydratation demeure faible (l'humidité relative variant entre 40 et $80 \mathrm{p} .100$ ) : la réaction de Maillard devient nulle aussi bien en milieu strictement anhydre qu'en solution diluée à l'extrême (Loncin et al., 1965).

En conséquence, la réaction de Maillard - bien que décelable dans les laits liquides - se développe avec une intensité particulière dans les laits en poudre, dans les sérums et dans tous les milieux lactés dont l'humidité est faible. Elle se manifeste dans des conditions différentes de celles où sont enregistrés les phénomènes de dénaturation physique.

La réaction de Maillard se traduit - sur le plan chimique par les faits suivants (Adrian, 1972) :

- un blocage et une destruction d'acides aminés qui entraînent une diminution de la qualité protidique, qu'elle soit mesurée in vitro ou in vivo,

- la formation de substances douées de propriétés antinutritionnelles et même toxiques, 
- l'apparition de molécules, volatiles ou non, exerçant une action psycho-sensorielle contribuant à la création d'arômes appréciés ou, au contraire, défavorables à la qualité commerciale.

En pratique, ce sont les répercussions sur la valeur protidique qui sont les manifestations les mieux étudiées de la réaction de Maillard. Elles se mesurent schématiquement selon deux grands principes :

- in vitro, en suivant l'évolution des acides aminés totaux ou, mieux, de leur fraction biologiquement utilisable (acide aminé disponible, ou digestible, ou utilisable, etc). Ces estimations portent habituellement sur la lysine seule. Cet acide aminé présente, en effet, une sensibilité 5 à 10 fois supérieure à celle des autres acides aminés indispensables lorsqu'une protéine est soumise à une réaction de Maillard (Adrian, 1967). Le comportement de la lysine constitue donc un indice très sensible de la réaction,

- in vivo, les effets de la réaction de Maillard s'estiment à la diminution de la digestibilité azotée ou bien de l'efficacité métabolique de l'azote (Valeur Biologique), ou encore en mesurant globalement les deux phénomènes en établissant le Coefficient d'Efficacité Protidique (C.E.P.) ou l'Utilisation Protidique Nette (U.P.N.), etc.

La réaction de Maillard peut se traduire différemment sur la digestibilité et l'utilisation azotées de la ration. La chute de la digestibilité est une manifestation constante de la réaction, traduisant le blocage ou la destruction des acides aminés ainsi que certains phénomènes secondaires.

Une diminution de la Valeur Biologique n'est pas obligatoirement observée après une réaction de Maillard: elle n'est enregistrée que dans le cas où la protéine alimentaire est limitée - initialement ou après chauffage - par son déficit en lysine. Si la lysine n'est pas le facteur-limitant de la protéine et si le traitement thermique n'est pas suffisant pour faire de cet acide aminé le facteur-limitant du produit chauffé, la Valeur Biologique demeurera inchangée (fig. 1).

Ce sera le cas, éventuellement, pour les protéines lactiques : la caséine est limitée par son déficit initial en méthionine et, à la suite d'un traitement léger, la réaction de Maillard n'aura pas détruit une quantité suffisante de lysine pour qu'elle présente un déficit supérieur à celui de la méthionine. Avec les protéines du sérum une situation analogue pourra être observée étant donné la richesse considérable de ces fractions en lysine.

En définitive, lorsqu'une protéine chauffée est soumise à une expérimentation animale, la réaction de Maillard provoquera toujours une diminution de la digestibilité azotée et - dans le cas d'une réaction intense - une baisse de la Valeur Biologique.

Enfin, parmi les différents produits alimentaires, le lait, le lactosérum et leurs dérivés lactosés sont parmi les plus sensibles à la 
Tendur en acides aminés

( en p.100 des protéines de $1^{\text {toeuf }}$ )

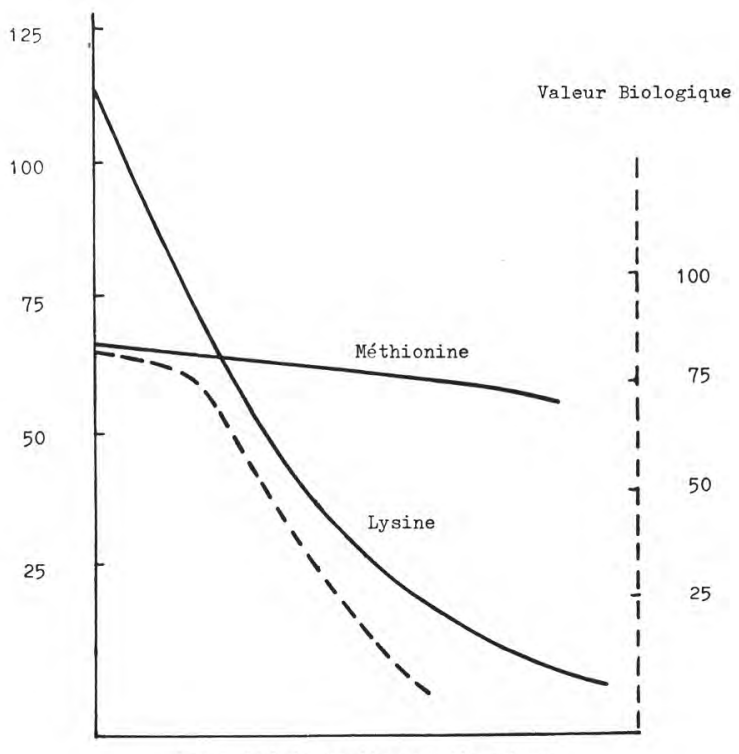

intensité du traitement thermique

fig. 1

Evolution de la qualité protidique du lait en fonction de l'intensité du traitement thermique : évolution des acides aminés facteurs limitant et de la Valeur Biologique.

réaction de Maillard (Adrian, 1967), cette situation résultant à la fois de la réactivité du lactose et de celle des protéines solubles du sérum.

\section{L'évolution de la qualité protidique dans les modèles expérimentaux}

Les modèles expérimentaux constitués au laboratoire offrent l'avantage de pouvoir préciser le rôle d'un facteur déterminé en n'étudiant qu'une seule variable. Leur utilité est incontestable pour la description théorique d'un phénomène.

Les modalités retenues pour ces études préliminaires diffèrent souvent, de façon profonde, des conditions dans lesquelles se déroulent un traitement industriel. Leur choix dépend, en fait, de la difficulté de reproduire à l'échelle du tube à essai l'intensité d'un phénomène observé lors d'une opération industrielle et aussi du but recherché qui oblige parfois à " caricaturer " les technologies appliquées aux matières alimentaires. 


\section{Réactivité du lactose}

Le lactose réagit avec les acides aminés grâce à sa fonction aldéhyde libre fixée sur la molécule de glucose, la constatation ayant été faite à maintes reprises (Mader et al., 1949 ; Cook, 1951, 1951 b ; Richards, 1963 ; Cheeke et Stangel, 1972 ; etc.).

Des études comparatives ont mis en évidence la très forte réactivité de ce disaccharide par rapport aux autres sucres simples. Lorsqu'on établit des réactions de Maillard et que les effets sont ramenés à des concentrations isomoléculaires, le lactose se révèle plus préjudiciable que le maltose ou le tréhalose et même plus actif que les hexoses en général (Schroeder et al., 1951 ; Frangne et Adrian, 1972).

En conséquence, la sensibilité des produits laitiers lors des traitements thermiques est due en premier lieu à leur forte concentration en lactose. Un produit lactosé introduit dans un mélange alimentaire va en accroître fortement la fragilité, ainsi que cela a été observé en étudiant le comportement thermique de biscuits lactés ou de pain au lait (Ehle et Jansen, 1965 ; Adrian et Frangne, 1969). L'élimination du lactose augmentera donc inévitablement la stabilité thermique des dérivés et des poudres lactiques.

\section{Réactivité des protéines}

Classiquement, l'intensité de la réaction de Maillard est d'abord déterminée par la nature du sucre mis en jeu. Néanmoins, la protéine exerce également un rôle dans la réaction puisque, toutes choses égales, lorsque la perte de lysine des albumines est de 40 p. 100 , celle des globulines est de 30 p. 100 , et celle des protéines céréalières se situe à 20 p. 100 environ (Frangne et Adrian, 1972).

Les protéines lactiques - jugées d'après le taux de destruction de leur lysine - se rangent indiscutablement parmi les molécules sensibles à la réaction de Maillard, l'affirmation s'appliquant particulièrement aux protéines du sérum. En effet, lorsque la perte moyenne de lysine, pour 20 protéines étudiées, est de 30 p. 100, les destructions enregistrées avec les protéines lactiques sont de 41 p. 100 pour la $\beta$ lactoglobuline, de 39 p. 100 pour la lactalbumine et de 30 p. 100 pour la caséine entière. Seule l'ovalbumine offre une plus grande fragilité (44 p. 100) que les protéines du lactosérum.

$\mathrm{Si}$ on juge la réaction de Maillard sur le comportement de la méthionine, les protéines lactiques offrent également une sensibilité assez marquée, surtout dans le cas des protéines du sérum. Dans des conditions drastiques, lorsque les protéines accusent une perte moyenne de 24 p. 100 de méthionine, le pourcentage de destruction est de 34 p. 100 pour la lactalbumine, de 31 p. 100 pour la $\beta$ lactoglobuline et seulement de 16 p. 100 pour la caséine entière. 
Mais, d'une façon générale, l'aspect le plus important de la réaction de Maillard demeure le comportement de la lysine. Par rapport à celui des autres acides aminés, il offre deux différences fondamentales :

- d'abord sa réactivité est intense en raison de son groupement $\varepsilon$ aminé qui réagit fortement avec les sucres,

- ensuite, le pourcentage de destruction de la lysine est lié significativement à la richesse de la protéine en lysine : la corrélation entre le p. 100 de destruction et le p. 100 de lysine dans la protéine est de + 0,60 (Frangne, 1972).

Ainsi, les protéines dont la lysine sera le plus endommagée par la réaction de Maillard seront celles qui sont le plus riches en cet acide aminé. En raison de leur richesse en lysine, toutes les fractions du lactosérum seront donc exposées à des risques importants lors des traitements technologiques.

\section{La perte de valeur protidique}

Un chauffage - en absence de sucres - est capable de provoquer des condensations inter-acides aminés qui les rendent inutilisables biologiquement (Evans et al., 1961 ; Bjarnason et Carpenter, 1970 ) et même de détruire des aminoacides à la suite de réactions de décarboxylation (Chatelus, 1964).

Cependant, ces phénomènes n'ont qu'une faible incidence pratique et il est licite d'assimiler à des manifestations de la réaction de Maillard les blocages et destructions d'acides aminés observés à la suite d'un chauffage en milieu complexe comportant des glucides.

Les effets de la réaction de Maillard sur la caséine brute sont connus depuis longtemps : la Valeur Biologique $\left({ }^{*}\right)$ est altérée par un traitement de $30 \mathrm{mn}$ à $140^{\circ} \mathrm{C}$ et une addition de lysine permet une restauration (Greaves et Morgan, 1933). Ce fait a été presque aussitôt attribué à un blocage de la lysine qui se trouvait alors sous une forme enzyme-résistante (Block et al., 1934).

C'est dire qu'empiriquement les conséquences nutritionnelles de la réaction de Maillard ont été observées depuis 40 ans !

Par la suite, en utilisant toujours des protéines lactiques plus ou moins purifiées, des confirmations et des précisions ont été fournies de divers côtés. D'abord, la destruction sélective de la lysine a été vérifiée aussi bien par Rao et al. (1963) que par Brüggemann et Erbersdobler (1968). Ces derniers, procédant à des analyses de caséine chauffée en présence de glucose, ont parfaitement démontré que les préjudices portaient sur les acides diaminés:

${ }^{*}$ ) Valeur Biologique : pourcentage de l'azote assimilé par la paroi intestinale qui est retenu par l'animal pour couvrir ses besoins azotés. 
Pourcentage de destruction des acides aminés après chauffage d'un mélange de caséine-glucose

\begin{tabular}{c|c|c}
\hline & $90^{\circ} \mathrm{C}$ & \multirow{2}{*}{$105^{\circ} \mathrm{C}$} \\
\hline Destruction moyenne des acides aminés & 3,5 & 8 \\
$-\quad$ de l'histidine & 17 & 17 \\
$-\quad$ de l'arginine & 12 & 22 \\
$-\quad$ de la lysine & 22 & 46 \\
\hline
\end{tabular}

Par ailleurs, la sensibilité des protéines lactiques a été confirmé indirectement : il suffit d'une purification incomplète de protéines aussi sensibles que celles du lactosérum pour que le lactose résiduel déclenche une réaction de Maillard importante au cours de traitements thermiques.

La démonstration en a été apportée par diverses équipes comme celle de Davis et al. (1949), Mader et al. (1949), Cook et al. (1951, $1951 \mathrm{~b}$ ), en utilisant des lactalbumines parfaitement purifiées ou contenant encore des traces de lactose ( 0,5 à 3,5 p. 100 environ).

En poussant les choses à l'extrême, une lactalbumine contenant de l'ordre de 3 p. 100 de lactose perd la totalité de sa valeur nutritionnelle après $1 \mathrm{~h}$ à $200^{\circ} \mathrm{C}$ en atmosphère sèche. Les animaux recevant un tel produit se comportent de façon identique à ceux nourris avec une alimentation sans azote (Davis et al., 1949).

Cependant, dans des chauffages plus classiques, on peut enregistrer une diminution de la digestibilité azotée sans pour autant que la Valeur Biologique des protéines en soit affectée (Chick et al., 1935; Mader et al., 1949 ; Schroeder et al., 1951 ; etc.). Ces faits ont été expliqués plus haut et la figure 1 rapporte l'évolution de la Valeur Biologique parallèlement à celle des acides aminés susceptibles de jouer le rôle de facteur-limitant.

En bref, grâce aux études théoriques conduites à l'aide de modèles expérimentaux, il est permis de déterminer les causes de la très grande fragilité thermique des produits laitiers.

\section{L'évolution de la qualité protidique dans les laits liquides}

Comme il a été précisé, la forte teneur en eau du lait constitue un facteur d'inhibition de la réaction de Maillard. Aussi, les altérations des laits liquides au cours des traitements thermiques doiventelles demeurer de faible ampleur dans les conditions habituelles de manipulations. 
Cette opinion est étayée aussi bien par l'examen de la composition en acides aminés des laits chauftés que par leur Valeur Biologique.

En ce qui concerne les acides aminés - totaux ou utilisables l'attention doit porter particulièrement sur la lysine en raison de la sensibilité sélective de cette molécule. Aussi, nous limiterons-nous généralement au devenir de cet acide aminé au cours des processus technologiques.

D'après Lang et al. (1965), la pasteurisation à $80^{\circ} \mathrm{C}$, la stérilisation ( $15 \mathrm{mn}$ à $\left.116^{\circ} \mathrm{C}\right)$, l'upérisation $\left(2,4 \mathrm{~s}\right.$ à $\left.150^{\circ} \mathrm{C}\right)$ n'entraînent pas une destruction significative d'acides aminés. Néanmoins, selon Payne-Botha et Bigwood (1959) et selon Dulcino et Lontie (1959), la stérilisation serait suffisamment sévère pour provoquer la destruction de 10 à 11 p. 100 de la lysine du lait.

En réalité, le préjudice nutritionnel résultant d'un traitement se détermine par le taux de blocage ou d'indisponibilité des acides aminés. Par rapport au lait cru, la perte de lysine utilisable est de 3 p. 100 dans le lait pasteurisé, de 8 p. 100 dans les poudres de lait atomisé (poudre spray), de 11 p. 100 dans le lait concentré sucré, de 12 p. 100 dans le lait sterilisé ; elle atteint 21 p. 100 dans le lait évaporé et environ 35 p. 100 dans les poudres séchées sur rouleaux ou sur cylindres dans des conditions normales (Mauron et al., 1955 ; Tsugo et al., 1961 ; Kisza et al., 1966 ; Ford et al., 1966 ; De Vuyst et al., 1972 ; etc.).

Ces valeurs moyennes reflètent le degré de sévérité des divers processus de stabilisation ou de transformation.

Comme les protéines du lait cru sont caractérisées par un excès de lysine de l'ordre de 10 p. 100 par rapport à la protéine de référence et par un déficit de 30 p. 100 en méthionine (Adrian, 1973), un blocage de lysine de l'ordre de 40 p. 100 ne doit pas entraîner une diminution de la Valeur Biologique, à la condition qu'il ne s'accompagne pas d'une aggravation du déficit de la méthionine, facteurlimitant. Il semble bien qu'il en soit ainsi dans la pratique : la Valeur Biologique ou le C.E.P. ne paraissent pas significativement altérés lorsque les protéines du lait constituent la seule source azotée de la ration et que l'on mesure les effets de la pasteurisation, du traitement UHT, de l'évaporation, de la condensation (Henry et al., 1944 ; Cook et al., 1951 c ; Schroeder et al., 1953 ; Karte, 1956 ; Gregory et al., 1964 ; Burton, 1965 ; Hostettler et al., 1965 ; etc.).

Seule la stérilisation classique entraîne une diminution d'environ 10 p. 100 de la Valeur Biologique ou du C.E.P. des protéines lactiques, que l'opération soit effectuée en présence d'oxygène ou sous gaz inerte (Cook et al., $1951 \mathrm{c}$; Schroeder et al., 1953 ; Burton, 1965).

L'ensemble de ces résultats - portant sur la valeur globale des protéines lactiques - ne doit pas masquer le préjudice relatif à la qualité de ces protéines : si le blocage de la lysine demeure insuffisant pour modifier la Valeur Biologique, il diminue cependant obli- 
gatoirement l'intérêt des laits chauffés en tant que source de lysine. Une comparaison entre un lait condensé et un lait évaporé a bien mis en relief les différences résultant d'une estimation globale des protéines ou de la mesure de leur qualité comme source de lysine: Le C.E.P. de ces deux produits est identique s'ils constituent la seule source azotée alimentaire mais diffère s'ils sont utilisés comme complément d'une ration céréalière (Mauron et Mottu, 1962) :

\begin{tabular}{l|c|c|c}
\hline & $\begin{array}{c}\text { Lysine } \\
\text { utilisable } \\
\text { (p. 100 } \\
\text { du lait cru) }\end{array}$ & $\begin{array}{c}\text { C.E.P. de la ration contenant } \\
10 \text { p. } 100 \\
\text { de protéines } \\
\text { de lait }\end{array}$ & $\begin{array}{c}\text { 6 p. 100 de protéines } \\
\text { de blé + 4 p. 100 de } \\
\text { protéines de lait }\end{array}$ \\
\cline { 2 - 3 } Lait condensé sucré & 97 & 3,59 & 2,81 \\
Lait évaporé & 78 & 3,59 & 2,42 \\
\hline
\end{tabular}

En dehors de la perte éventuelle de lysine, les chauffages industriels du lait ne semblent pas etre responsables d'action préjudiciable sur le plan nutritionnel. En particulier, si on retient comme critère le taux d'azote total solubilisé, la protéolyse in vitro n'est pas entravée à la suite d'un traitement thermique (Dulcino et Lontie, 1959 ) et elle pourrait même en être facilitée dans certains cas (Schroeder et al., 1953). Il conviendrait cependant de pratiquer des tests plus fins, notamment de suivre la libération des acides aminés les plus importants pour pouvoir porter un jugement définitif.

On peut ajouter que pendant les phases de conservation, la qualité protidique des laits liquides n'est pas endommagée de façon significative après 6 mois (Hugot et Causeret, 1962) ou même 1 an à la température ordinaire (Cook et al., $1951 \mathrm{c}$ ). Des conditions tout à fait exceptionnelles sont nécessaires pour que la conservation entraîne une diminution de la qualité azotée : par exemple, un stockage pendant 8 années bloque les $2 / 3$ de la lysine d'un lait évaporé, c'est-à-dire préalablement concentré (Tsugo et al., 1961).

En conclusion, le lait liquide - c'est-à-dire ayant conservé l'intégrité de son eau - se trouve pratiquement à l'abri des effets thermiques, les processus industriels n'endommagent que dans une faible mesure son potentiel protidique.

Cette stabilité - due à la forte dilution du lait - s'oppose à la thermo-sensibilité de produits comme les poudres de lait ne renfermant qu'une faible humidité résiduelle. 